\title{
WiMAX, LTE, and WiFi Interworking
}

\author{
Rashid A. Saeed, ${ }^{1}$ Ahmed A. M. Hassan Mabrouk, ${ }^{2}$ Amitava Mukherjee, ${ }^{3}$ \\ Francisco Falcone, ${ }^{4}$ and K. Daniel Wong ${ }^{5}$ \\ ${ }^{1}$ Department of Electrical and Computer Engineering, Engineering Faculty, IIUM, Kuala Lumpur, Malaysia \\ ${ }^{2}$ Information and Communication Technology Faculty, IIUM, Kuala Lumpur, Malaysia \\ ${ }^{3}$ IBM India Private Limited, Salt Lake, Calcutta 700 091, India \\ ${ }^{4}$ EE Department, Universidad Pública de Navarra, Campus de Arrosadía, Pamplona, 31006 Navarre, Spain \\ ${ }^{5}$ Daniel Wireless LLC, Palo Alto, CA 94306, USA
}

Correspondence should be addressed to Rashid A. Saeed, eng_rashid@ieee.org

Received 28 April 2010; Accepted 28 April 2010

Copyright (c) 2010 Rashid A. Saeed et al. This is an open access article distributed under the Creative Commons Attribution License, which permits unrestricted use, distribution, and reproduction in any medium, provided the original work is properly cited.

Recently wireless network interworking has become an important area of research in academia and industry. This is due to the huge diversity of wireless network types, which range from wireless body area network (WBAN) covering areas up to a few inches to wireless regional area networks (WRANs) covering up to several miles. All these types of networks have been developed separately with different usage and applications scenarios, which make interworking between them a challenging task.

The main challenges in wireless interworking of connecting the cellular network with the other wireless networks include issues like security, seamless handover, location and emergency services, cooperation, and QoS. The developed interworking mechanisms, that is, unlicensed mobile access (UMA), IP Multimedia Subsystem (IMS), and Media independent handover $(\mathrm{MIH})$, due to the characteristics of wireless channel, need to be analyzed and tested under various circumstances.

The aim of this special issue in Journal of Computer Systems, Networks, and Communications (JCSNC) is to highlight the problems and emphasize and analyze the solutions in this area, which can give a guideline to telecom industry for new techniques and business opportunities. Many researchers from different parts of the world and different background have participated in the issue. The accepted papers are diverse at different interworking levels, spanning from network layer down to link level for example, the paper entitled "Technology-integration framework for fast and low cost handovers, case study: WiFiWiMAX network" by M. Kassab, et al.where the end-to-end delay is optimized with minimum management signaling cost.

On the other hand, in "WiFi and WiMAX secure deployments" by P. Trimintzios and G. Georgiou, the security intrusion that may occur during handover is discussed. In the paper "Seamless video session handover between WLANs" by C. C. Monteiro, et al. an architecture for session proxy (SP) with video streaming quality preservation has been developed.

At the physical layer, the paper "Investigation of cooperation technologies in heterogeneous wireless networks" by Z. Sun and W. Wang discussed the radio access technology (RAT) for various standards, where issues like multiradio resource management (MRRM) and generic link layer (GLL) were proposed. In the paper entitled "A multi-standard frequency offset synchronization scheme for $802.11 \mathrm{n}, 802.16 \mathrm{~d}$, LTE and DVB-T/H systems" by J. González-Bayón et al. carrier frequency offset in OFDM systems is discussed where common synchronization structure for all these systems is proposed.

C. So-In et al. in "Capacity Evaluation for IEEE 802.16e Mobile WiMAX" emphasize on the overhead of the WiMAX protocol and its effect on the link capacity. Many applications have been tested that is, Mobile TV and VOIP. In the same area P. T. T. Pham and T. Wada's paper "Effective scheme of channel tracking and estimation for mobile WiMAX DLPUSC System" discussed the packet error rate (PER) and user throughput in various channels.

K.-P. Lin and H.-Y. Wei discussed a new random walk mobility model in "Paging and location management in IEEE 
$802.16 j$ multihop relay network". The proposed model is suitable for multihop relay network, where the handover process is frequently performed.

Finally, "Multi mode flex-interleaver core for baseband processor platform" by R. Asghar and D Liu introduces a new flexible interleaver architecture supporting many standards like WLAN, WiMAX, HSPA+, LTE, and DVB at the system level. Both maximum flexibility and fast switchability were examined during run time.

This special issue would not have come true without the tight guidelines and support from the Editor-in-Chief Professor Hsiao-Hwa Chen and Mariam Albert the editorial staff in Hindawi Publishing Corporation.

Rashid A. Saeed Ahmed A. M. Hassan Mabrouk Amitava Mukherjee Francisco Falcone K. Daniel Wong 

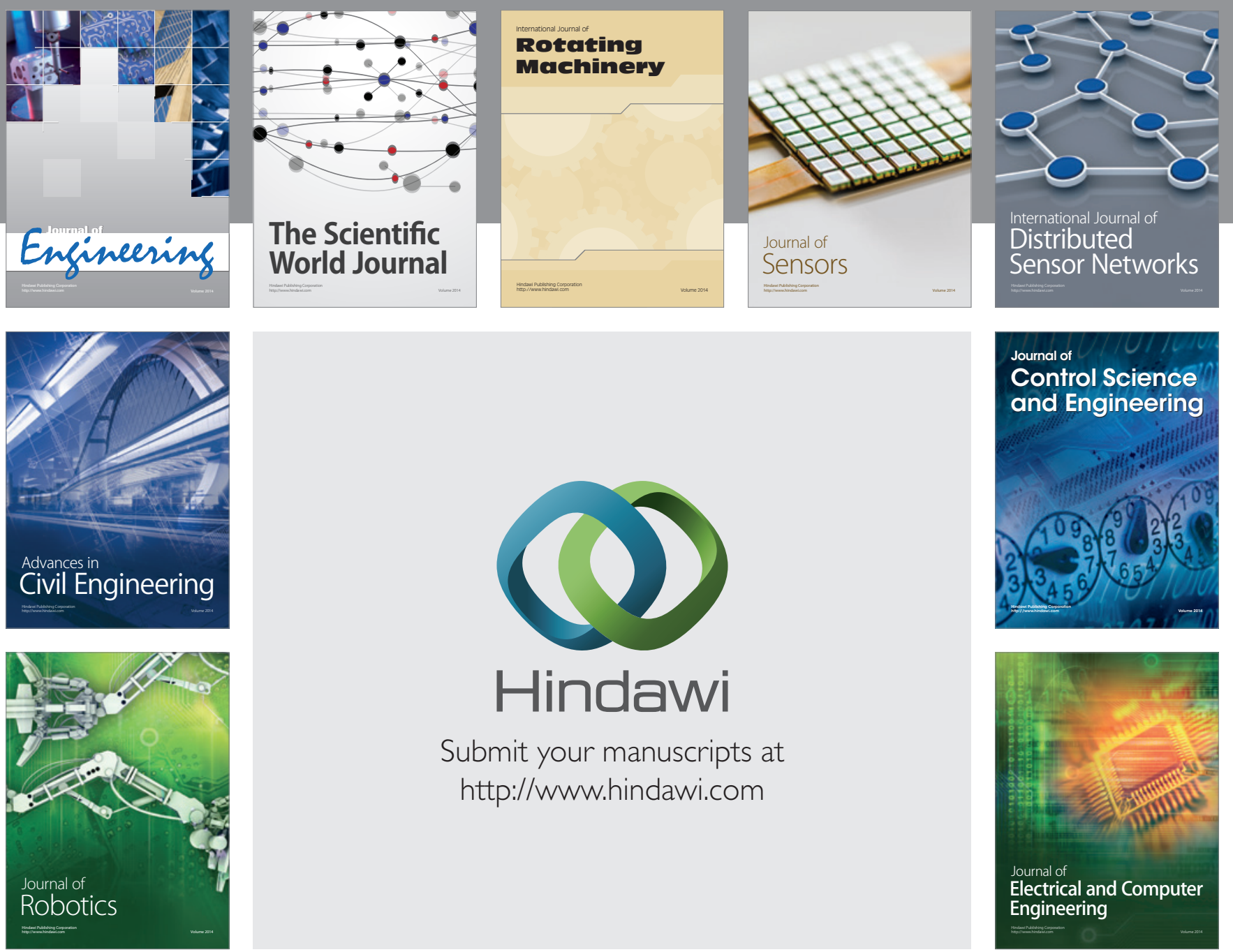

Submit your manuscripts at

http://www.hindawi.com
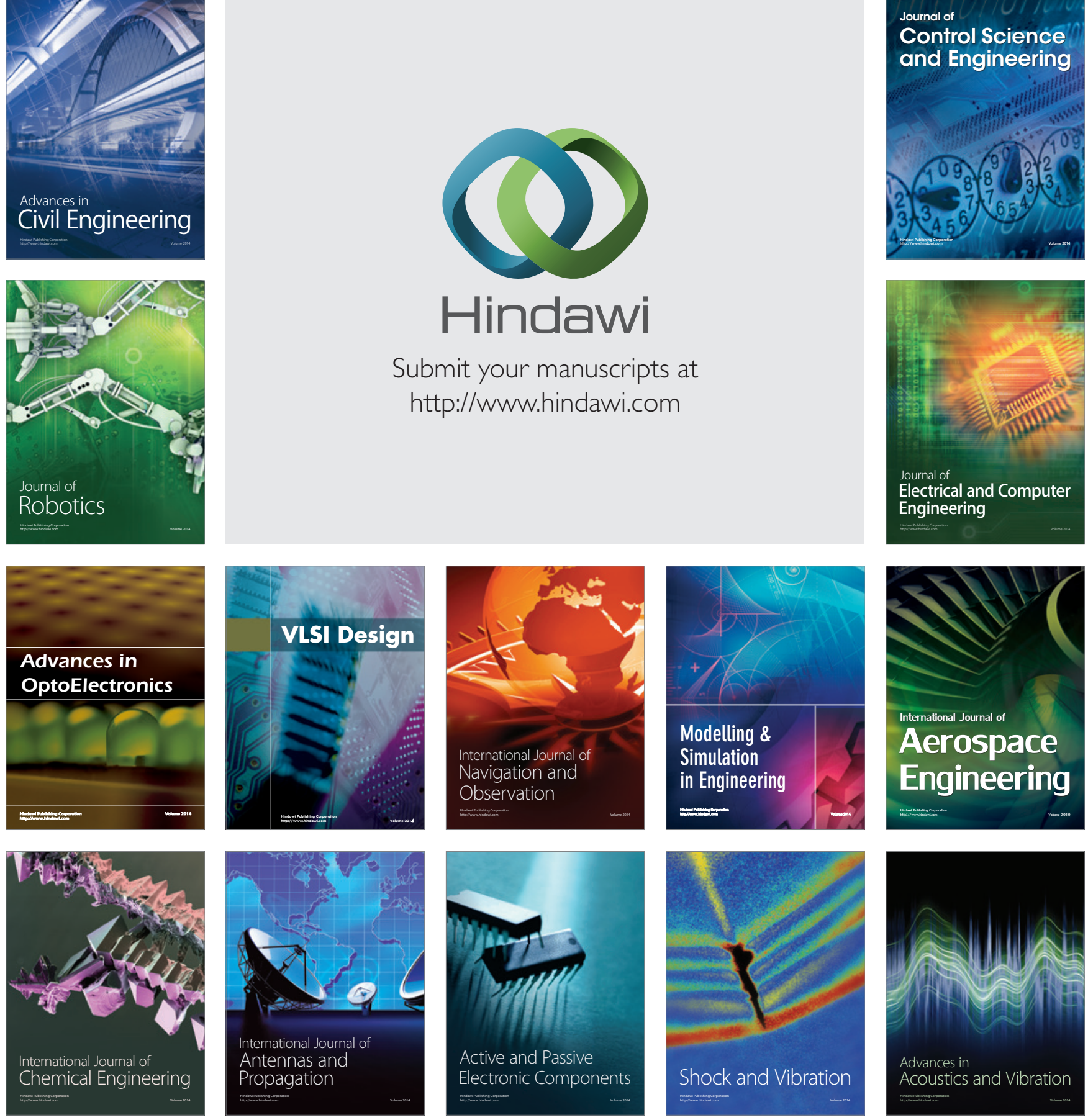